\title{
Effects of electroacupuncture on apoptotic pathways in a rat model of focal cerebral ischemia
}

\author{
YU RI KIM ${ }^{1}$, HA NEUI KIM ${ }^{1}$, JI YEON JANG ${ }^{1}$, CHEOL PARK $^{2}$, JUN HYUK LEE ${ }^{3}$, \\ HWA KYOUNG SHIN $^{1}$, YUNG HYUN CHOI ${ }^{2}$ and BYUNG TAE CHOI ${ }^{1}$ \\ ${ }^{1}$ Division of Meridian and Structural Medicine, School of Korean Medicine, Pusan National University, \\ Yangsan 626-870; ${ }^{2}$ Department of Biochemistry, College of Oriental Medicine, Anti-Aging Research Center \\ and Blue-Bio Industry RIC, Dongeui University, Busan 614-052; ${ }^{3}$ Chemistry and Biotechnology \\ Examination Bureau, Korean Intellectual Property Office, Daejeon 302-701, Republic of Korea
}

Received June 26, 2013; Accepted September 18, 2013

DOI: $10.3892 / \mathrm{ijmm} .2013 .1511$

\begin{abstract}
In this study, we investigated the molecular mechanisms underlying the anti-apoptotic properties of electroacupuncture (EA) in a rat model of middle cerebral artery occlusion (MCAO). Treatment with $2 \mathrm{~Hz}$ EA (1 mA) resulted in a markedly reduced infarct area after stroke, particularly in the middle region of the brain. Treatment with EA resulted in a significant decrease in the number of apoptotic cells identified by Hoechst 33342 and TUNEL staining. According to the results of the analysis for proteins involved in apoptosis, treatment with EA resulted in a significantly reduced expression of death receptor (DR)5. Among the members of the Bcl-2 family, a higher expression of anti-apoptotic Bcl-2 and Bcl-xL was observed in the rats treated with EA, compared with the untreated rats with MCAO. As regards the expression of the inhibitor of apoptosis protein (IAP) family, a higher expression of anti-apoptotic cIAP-1 and -2 was also detected in the cortex of the EA-treated rats. Using western blot analysis, we observed that activated caspase- 3 was only significantly arrested by EA treatment in the rats with MCAO; however, according to the results of the caspase assay, the activities of caspase- $3,-8$ and -9 were markedly inhibited by EA treatment. These results suggest that treatment with EA exerts anti-apoptotic effects in cerebral ischemia in a rat model of MCAO and that these effects are
\end{abstract}

Correspondence to: Dr Byung Tae Choi, Division of Meridian and Structural Medicine, School of Korean Medicine, Pusan National University, Yangsan 626-870, Republic of Korea

E-mail: choibt@pusan.ac.kr

Dr Yung Hyun Choi, Department of Biochemistry, College of Oriental Medicine, Anti-Aging Research Center and Blue-Bio Industry RIC, Dongeui University, Busan 614-052, Republic of Korea E-mail: choiyh@deu.ac.kr

Key words: electroacupuncture, apoptosis, cerebral ischemia, death receptor, Bcl-2, caspase associated with the inhibition of the DR and mitochondrial apoptotic pathways.

\section{Introduction}

Stroke is regarded as the second most common cause of mortality worldwide (1). Tissue plasminogen activator for removal of the thrombus, which must be administered during the early stages, within 4-5 h of stroke onset, is the currently approved pharmacotherapy administered after ischemic stroke (1-3). Reperfusion following cerebral ischemia plays an important role in the recovery of blood flow in order to reduce neuronal damage; however, cerebral ischemia and reperfusion trigger multiple cell signaling pathways, leading to cell survival or cell damage (4).

Accumulating evidence suggests that, apart from necrosis, apoptosis is an additional cause of hypoxic-ischemic neuronal loss in the nervous system. The ischemic core undergoes necrotic injury within minutes after ischemic stroke; however, the ischemic penumbra, the border of the necrotic core, undergoes apoptosis within several hours or days with the activation of multiple death pathways $(5,6)$. Neuronal cells in the distal penumbra area tolerate a longer duration of ischemia, compared with those of the core, and cerebral blood flow is less severely affected (7). Damage to the penumbra caused by ischemic stroke may be reversible, comprising an energydependent apoptosis when blood flow is restored $(5,8)$.

Due to the contribution of neuronal cell loss, apoptosis is a fundamental target for neuroprotective strategies in the management of cerebral ischemia $(9,10)$. The overload of intracellular calcium by excessive overstimulation of ionotropic glutamate receptors following cerebral ischemia can cause irreversible neuronal cell death through intracellular apoptotic signaling cascades (11-13). Calpain, caspases, phospholipase A2 and endonucleases, calcium-dependent effective proteins, lead to cell death $(14,15)$.

The major apoptotic signaling pathways may be classified as the extrinsic death receptor (DR) and intrinsic mitochondrial apoptotic pathways (4). DR apoptotic pathways are regulated by the activation of DRs with their ligands on the cell membrane, finally resulting in the activation of caspases (16). 
The mitochondrial apoptotic pathway is mediated by the activation of pro-apoptotic proteins, such as Bid and Bax, and Bcl-2 family proteins (17). Both the extrinsic and intrinsic apoptotic pathways play a vital role in the execution of cell death, recruiting downstream apoptotic molecules (4).

Acupuncture, a traditional therapeutic treatment in Oriental medicine, has long been used in clinical practice for the prevention and treatment of stroke. In particular, electroacupuncture (EA), which involves the application of electrical stimulation with acupuncture needles, has been widely used both in clinical practice and in studies using animal stroke models in Korea. Accumulating evidence has indicated that treatment with EA can effectively attenuate cerebral infarction and exerts anti-apoptotic and/or neuroprotective effects after hypoxic-ischemic insults $(3,18-20)$.

The regulation of apoptotic signaling pathways following focal or global ischemia may result in the attenuation of neuronal cell death and may lead to the development of novel therapeutic strategies for the treatment of cerebral ischemia. Treatment with EA may reverse the damage caused to the ischemic penumbra by inhibiting the activation of apoptotic pathways; however, the underlying mechanisms remain unclear. According to our results, treatment with EA at the acupoints corresponding to Baihui and Qihai resulted in a significantly reduced infarct volume and improvement of neurological outcome after stroke, consistent with previous studies $(21,22)$. Therefore, we hypothesized that treatment with EA can alleviate cell death resulting from ischemic injury through the main routes of the apoptotic pathway, particularly in the cortex of the brain, which tolerates a longer duration of ischemia, which may be reversible by treatment with EA. We investigated the apoptotic signaling pathways involved in the beneficial effects of EA in a rat model of middle cerebral artery occlusion (MCAO).

\section{Materials and methods}

Antibodies. DR4, DR5, tumor necrosis factor-related apoptosis-inducing ligand (TRAIL), Fas, Fas ligand (FasL), Bcl-2, Bcl-xL, Bax, Bad, Bid, X-linked inhibitor of apoptosis protein (XIAP), caspase-3, caspase-8, caspase-9, poly[ADP-ribose] polymerase (PARP)-1, $\beta$-catenin and phospholipase $\mathrm{C} \gamma 1$ (PLC $\gamma 1$ ) and secondary antibodies were supplied by Santa Cruz Biotechnology (Santa Cruz, CA, USA). cIAP-1 and cIAP-2 were supplied by Calbiochem (San Diego, CA, USA).

Animals. Male Sprague-Dawley rats averaging 150-180 g in weight were obtained from DooYeol Biotech (Seoul, Korea). The rats were housed at $22^{\circ} \mathrm{C}$ under alternating $12 \mathrm{~h}$ cycles of dark and light, and were fed a commercial diet and allowed access to tap water ad libitum, commencing one week before the study began and continuing throughout the study. All experiments were approved by the Pusan National University Animal Care and Use Committee in accordance with the National Institutes of Health Guidelines. Each group included six rats and all treatments were administered under isoflurane (Choongwae, Seoul, Korea) anesthesia, using a VIP 3000 calibrated vaporizer (Midmark, Orchard Park, OH, USA). The rats were randomly assigned to the sham-operated, MCAO and $\mathrm{MCAO}+\mathrm{EA}$ groups. The rats in the MCAO group were subjected to MCAO only and the rats in the MCAO + EA group received EA stimulation immediately after reperfusion. The sham-operated rats underwent the same procedure without MCAO.

Focal cerebral ischemia. Focal cerebral ischemia was induced by occluding the middle cerebral artery using the intraluminal filament technique. Rats used in the experiment were anesthetized using $2.0 \%$ isoflurane (Choongwae) and cerebral blood flow was monitored using a PeriFlux System 5000 laser-Doppler flowmeter (Perimed, Stockholm, Sweden). Under a SXZ16 operating microscope (Olympus, Tokyo, Japan), the right external carotid artery and the internal carotid artery were bound with 4-0 silk sutures (Mersilk; Ethicon Inc., Somerville, NJ, USA). The middle cerebral artery was occluded by a 2.0-3.0 cm length of 4-0 nylon suture with a silicon-coated tip. After $90 \mathrm{~min}$ of MCAO, the filament was withdrawn and reperfusion was confirmed using laser Doppler examination.

EA stimulation. Under light isoflurane anesthesia, two bilateral stainless-steel $0.2 \mathrm{~mm}$-diameter needles were inserted to a depth of approximately $3 \mathrm{~mm}$ at the acupoints corresponding to Baihui (GV20, the midpoint of the line connecting the apexes of both ears on the parietal bone) and Qihai (CV6, the lower abdomen and on the anterior midline) in men, and were connected to a Pulsemaster Multi-channel Stimulator SYS-A300 electrical stimulator (World Precision Instruments, Berlin, Germany). EA treatment was performed with $2 \mathrm{~Hz}$ stimulation for $30 \mathrm{~min}$ and intensity was set at $1 \mathrm{~mA}$. EA was administered twice per day at 12-h intervals immediately after occlusion. Animals that did not receive EA were subjected to the same anesthesia as those that received EA.

Determination of infarct area. For quantification of ischemic damage, the rats were anesthetized by an intraperitoneal injection of $8 \%$ chloral hydrate solution $(300 \mathrm{mg} / \mathrm{kg})$ and the areas of cerebral infarction were evaluated by 2,3,5-triphenyltetrazolium chloride (TTC; Sigma-Aldrich, St. Louis, MO, USA) staining three days after occlusion. The brains were cut into eight 2-mm-thick sections and stained with $2 \%$ TTC in phosphate-buffered saline (PBS) for $30 \mathrm{~min}$ at room temperature. The sections were then stored in formaldehyde solution (Duksan Pure Chemicals, Ansan, Korea). Photographs of each brain section were acquired using a digital camera by arranging the sections in turn and the infarct area was measured using an I-Solution ${ }^{\mathrm{TM}}$ Image Analyzer [Image \& Microscope Technology (IMT), Vancouver, BC, Canada]. The infarct area was calculated by the direct measurement of the areas of each section.

Measurement of apoptotic neuronal death. The rats received intracardial perfusion with $200 \mathrm{ml}$ of PBS followed by $100 \mathrm{ml}$ of fixative containing 4\% paraformaldehyde in PBS under chloral hydrate anesthesia and the cerebral cortex of the ipsilateral ischemic area was removed. The removed tissue was kept in the same fixative for $24 \mathrm{~h}$, followed by immersion in $30 \%$ sucrose for $48 \mathrm{~h}$ at $4^{\circ} \mathrm{C}$ for cryoprotection. Frozen sections (14- $\mu$ m-thick) were then prepared. Apoptotic neuronal death was characterized by staining with Hoechst 33342 (Invitrogen, 
Carlsbad, CA, USA) and a terminal deoxynucleotidyl transferase-mediated dUTP nick end-labeling (TUNEL) assay. Tissues were stained with Hoechst 33342 at room temperature for $30 \mathrm{~min}$ and fragmented or condensed DNA was counted. TUNEL assays were performed using a Fluorometric TUNEL System (Promega, Madison, WI, USA) and TUNEL-positive cells were counted. Quantitative analysis was performed in a blinded manner by counting the number of apoptotic cells in five random regions using a fluorescence microscope (Carl Zeiss, Göttingen, Germany). Data are presented as the total number of apoptotic cells.

Western blot analysis. Cortical tissues were washed in cold HEPES buffer and homogenized in lysis buffer [200 $\mathrm{mM}$ Tris (pH 8.0), 150 mM NaCl, 2 mM EDTA, 1 mM NaF, $1 \%$ NP40, $1 \mathrm{mM}$ phenylmethanesulfonylfluoride (PMSF), $1 \mathrm{mM}$ $\mathrm{Na}_{3} \mathrm{Vo}_{4}$ and protease inhibitor cocktail]. Equal amounts of protein were then separated by $10 \%$ sodium dodecyl sulfatepolyacrylamide gel electrophoresis (SDS-PAGE); the resolved proteins were then transferred onto nitrocellulose membranes (Whatman, Dassel, Germany). The membranes were incubated with antibodies overnight at $4^{\circ} \mathrm{C}$. The membranes were subsequently incubated with horseradish peroxidaseconjugated secondary antibody. $\beta$-actin was used as a loading control for all experiments. Densitometric analysis was performed using an ImageQuant LAS-4000 imaging system (Fujifilm, Tokyo, Japan) for the quantification of immunoreactivity corresponding to the bands.

Caspase assay. Colorimetric assay kits (R\&D Systems, Minneapolis, MN, USA), which utilize synthetic tetrapeptides [Asp-Glu-Val-Asp (DEAD) for caspase-3; Ile-Glu-Thr-Asp (IETD) for caspase-8; and Leu-Glu-His-Asp (LEHD) for caspase-9] labeled with p-nitroaniline (pNA), were used for the determination of caspase activity. Briefly, $150 \mu \mathrm{g}$ protein in the cerebral cortex were mixed with extraction buffer $[40 \mathrm{mM}$ HEPES (pH 7.4), 20\% glycerol (v/v), 1 mM EDTA, 0.2\% NP-40 and $10 \mathrm{mM}$ DL-DTT] containing $100 \mu \mathrm{M}$ fluorogenic peptide substrate, diluted with extraction buffer setting a volume of $100 \mu \mathrm{l}$ per sample in a microtiter plate, followed by incubation at $37^{\circ} \mathrm{C}$ for $2 \mathrm{~h}$ in the dark. For the determination of caspase activity, changes in absorbance were measured at a wavelength of $405 \mathrm{~nm}$ using an ELISA reader.

Data analyses. All data are expressed as the means \pm SEM and the SigmaStat statistical program Version 11.2 (Systat Software, San Jose, CA, USA) was used for data analysis. For statistical analysis of the data, the Student's t-test was used when comparing two groups, and one-way ANOVA via Tukey's post hoc comparison was used when comparing more than two groups. A p $<0.05$ was considered to indicate a statistically significant difference.

\section{Results}

Effects of EA on the infarct area. We performed EA on the rats with $\mathrm{MCAO}$ at vessel reperfusion and $12 \mathrm{~h}$ after occlusion twice the first day and twice per day at 12-h intervals thereafter. To determine which region of the brain shows beneficial effects by EA, we analyzed the infarct area of each

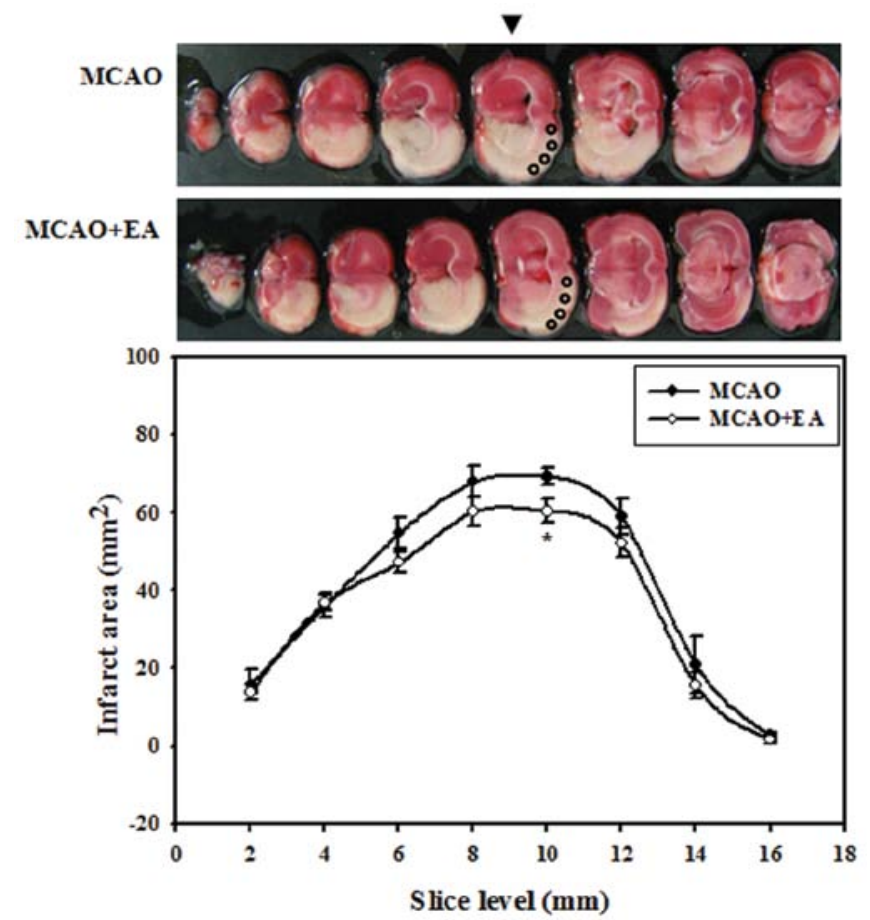

Figure 1. Effects of electroacupuncture (EA) on the infarct area at slice level of the brain three days after occlusion. The graph shows the infarct area at each coronal slice level and pale colored regions in the $2 \%$ TTC-stained brain indicate ischemic portions of the brain. EA treatment resulted in a reduction of the infarct area mainly in the middle cortical regions, particularly at the fifth slice level. The arrow head indicates the fifth slice level of the brain showing the cortical regions for which histological observation, measurement of apoptotic death and western blot analysis were performed. Data are presented as the means \pm SEM. ${ }^{*} \mathrm{p}<0.05$ vs. MCAO group rats; $n=6$ in each group. $\mathrm{MCAO}$, middle cerebral artery occlusion.

coronal slice between the rats in the MCAO and MCAO + EA group three days after occlusion. Treatment with EA resulted in the reduction of the infarct area from the anterior region to the posterior region of the brain. In addition, a significant decrease in the infarct area was observed at the middle region of the brain, mainly in the fifth slice level at $10 \mathrm{~mm}$ in distance from the frontal pole. These results suggested that EA stimulation exerted a significant neuroprotective effect against focal cerebral ischemia, particularly in the middle region of the brain. We used cortical tissue obtained from the fifth slice level within circles measuring $2 \mathrm{~mm}$ in diameter for the analysis of apoptosis (Fig. 1).

Effects of EA on apoptotic cell death. We performed Hoechst 33342 and TUNEL staining as a marker of apoptotic cells. The cells with fragmented or condensed DNA and TUNEL-positive cells were counted; the data are presented as apoptotic neurons as a percentage of total neurons. The number of apoptotic cells significantly increased at 5 and $12 \mathrm{~h}$ after occlusion in the rats with MCAO, compared with the sham-operated rats. However, the number of apoptotic cells markedly decreased following EA stimulation (Fig. 2). These changes indicate that progressive cell loss in the cerebral cortex of an ischemic cerebrum may occur due to apoptosis, not necrosis, and may be alleviated by EA stimulation. 


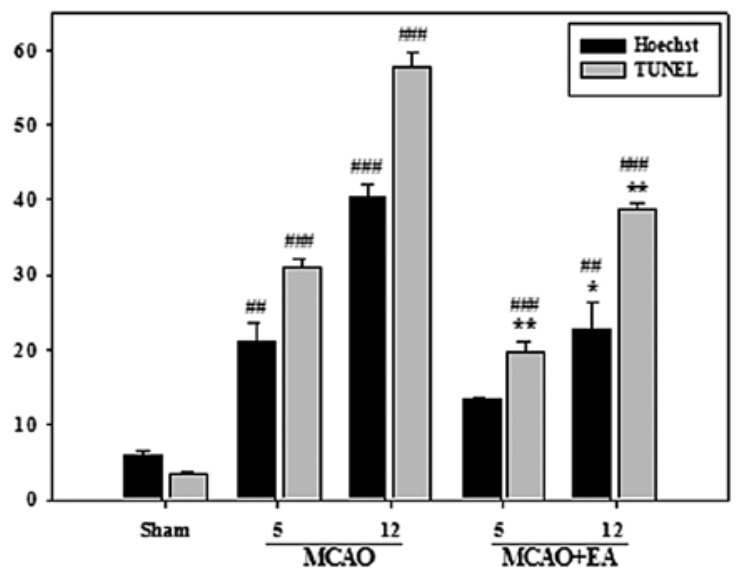

Hours af ter occlusion

Figure 2. Apoptotic analysis by Hoechst 33342 and TUNEL staining in the cerebral cortex of the brain. The number of apoptotic cells markedly increased in the MCAO group rats, compared with the sham-operated rats; however, these changes were markedly inhibited by treatment with EA. ${ }^{\# \#}$ p $<0.01$ and $\#$ \#\# $<0.001$ vs. sham-operated rats; ${ }^{*} \mathrm{p}<0.05$ and ${ }^{* *} \mathrm{p}<0.01$ vs. MCAO group rats; $n=6$ in each group. EA, electroacupuncture; $\mathrm{MCAO}$, middle cerebral artery occlusion
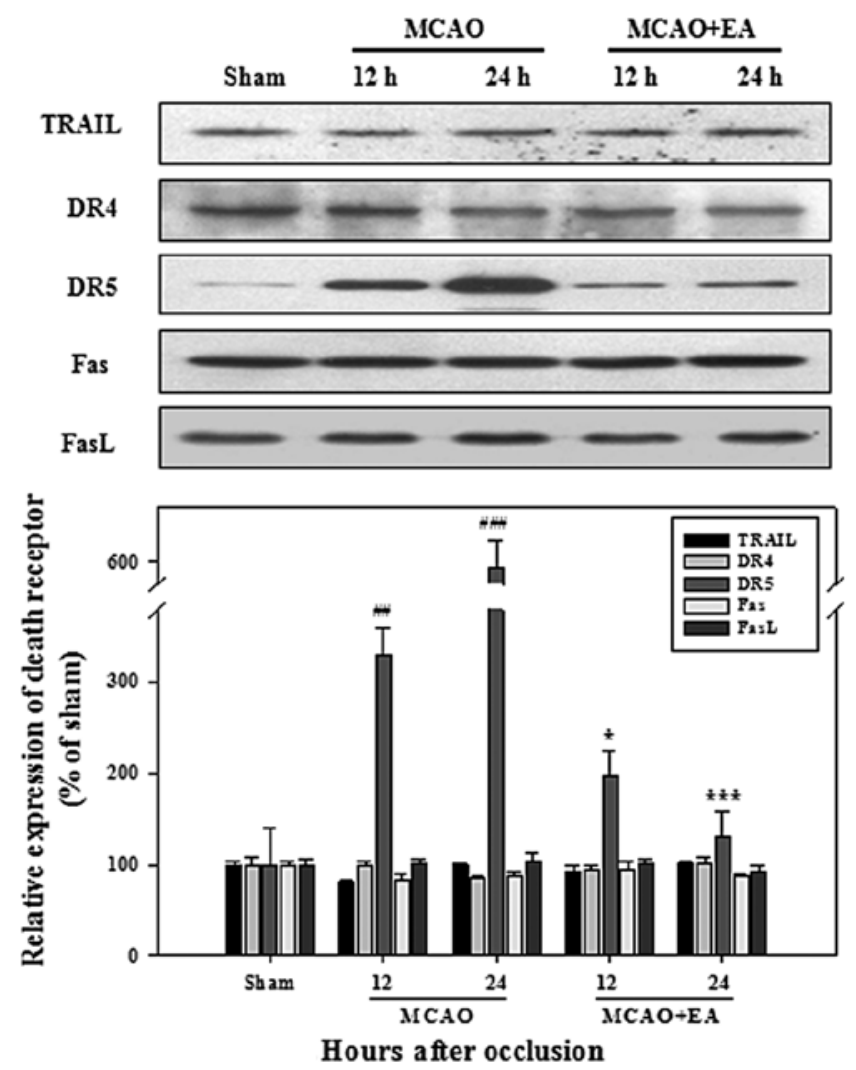

Figure 3. Western blot analysis and densitometric analysis for death ligands and receptors in the cerebral cortex of the brain. Each subunit is expressed as a percentage of the sham-operated group. Representative results results from three independent experiments are shown. The expression of DR5 in the MCAO group rats was significantly inhibited by treatment with EA. ${ }^{\# \#} \mathrm{p}<0.01$

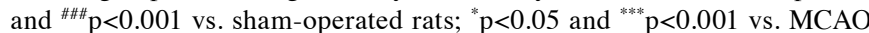
group rats; $n=3$ in each group. EA, electroacupuncture; MCAO, middle cerebral artery occlusion; DR5, death receptor 5 .

Effects of EA on apoptotic signaling pathways. We performed western blot analysis to determine whether neuronal apoptosis

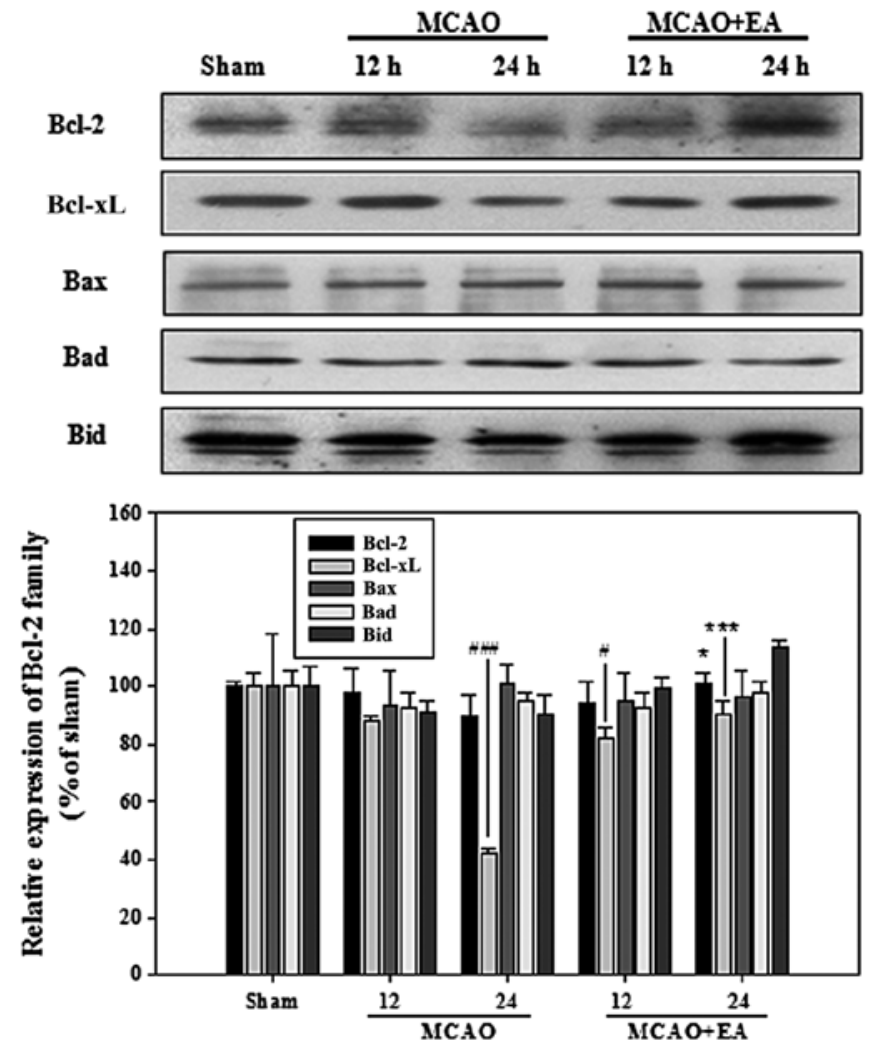

Hours a fter occlusion

Figure 4. Western blot analysis and densitometric analysis for Bcl-2 family members in the cerebral cortex of the brain. Each subunit is expressed as a percentage of the sham-operated group. Representative results results from three independent experiments are shown. The expression of Bcl-2 and $\mathrm{Bcl}-\mathrm{XL}$ in the rats in the MCAO + EA group showed a marked increase, compared with the MCAO group rats. ${ }^{\#} \mathrm{p}<0.05$ and ${ }^{\# \# \#} \mathrm{p}<0.001 \mathrm{vs}$. sham-operated rats; ${ }^{*} p<0.05$ and ${ }^{* * *} \mathrm{p}<0.001$ vs. MCAO group rats. $\mathrm{n}=3$ in each group. EA, electroacupuncture; MCAO, middle cerebral artery occlusion.

occurs through the death receptor and mitochondrial pathways. As regards the expression of death ligands and receptors, no significant changes were observed between the rats in the $\mathrm{MCAO}$ and MCAO + EA group, apart from DR5. The expression of DR5 was significantly higher in the rats with MCAO, compared with the sham-operated rats at 12 and $24 \mathrm{~h}$ after occlusion; however, this increase was reversed following treatment with EA (Fig. 3). Among the Bcl-2 family members, only the expression of $\mathrm{Bcl}-\mathrm{xL}$ showed a significant decrease in the rats with MCAO; however, a higher expression of Bcl-2 and $\mathrm{Bcl}-\mathrm{xL}$ was observed in the rats in the MCAO + EA group, compared with the MCAO group rats at $24 \mathrm{~h}$ after occlusion (Fig. 4). The IAP family in the rats with MCAO exhibited a lower expression compared with the sham-operated rats; however, a significantly higher expression of cIAP-1 and cIAP-2 was observed in the rats in the MCAO + EA group, when compared with the rats in the MCAO group (Fig. 5). We observed only one instance of activated caspase-3 expression, which was significantly arrested by treatment with EA in the rats with MCAO (Fig. 6A). Cleaved PLC $\gamma 1$ in the rats with MCAO was markedly inhibited by treatment with EA and a slightly lower total PARP expression was observed in the rats with MCAO, compared with the rats in the MCAO + EA group 

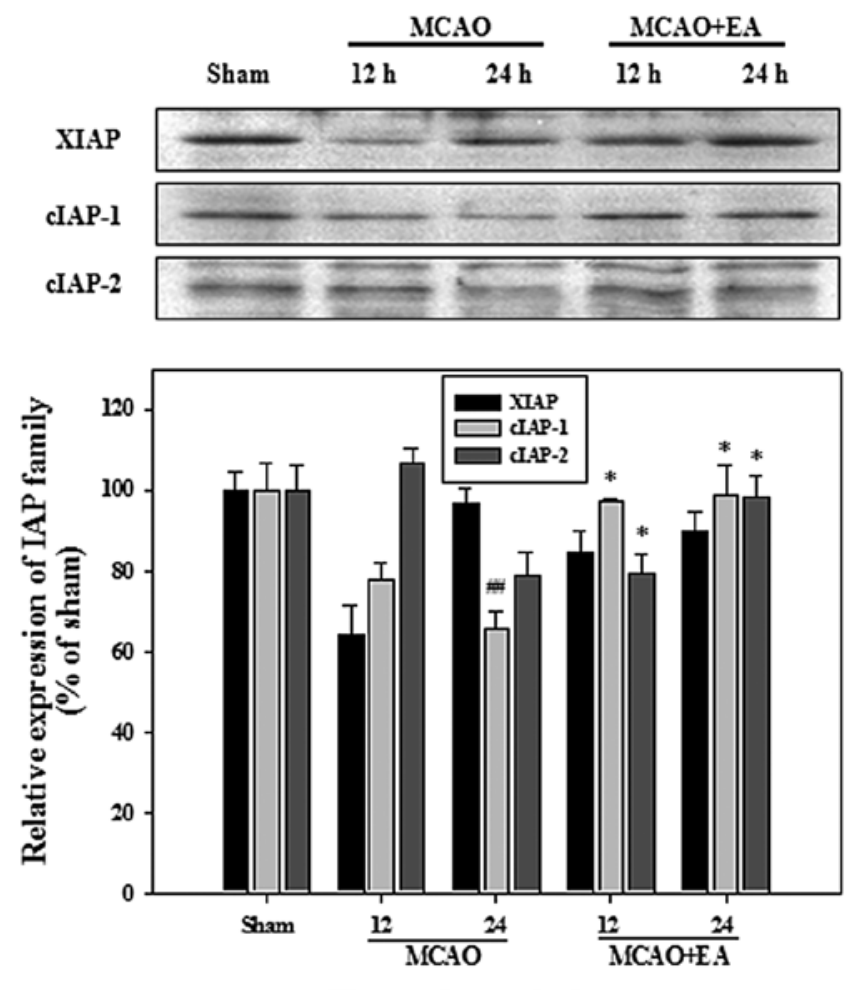

Hours after occlusion

Figure 5. Western blot analysis and densitometric analysis for IAP family members in the cerebral cortex of the brain. Each subunit is expressed as a percentage of the sham-operated rats. Representative results results from three independent experiments are shown. The expression of cIAP-1 and cIAP- 2 in the rats in the $\mathrm{MCAO}+\mathrm{EA}$ group showed a marked increase, compared with MCAO group rats. ${ }^{\# \#} \mathrm{p}<0.01$ vs. sham-operated rats; " $\mathrm{p}<0.05$ vs. MCAO group rats; $n=3$ in each group. EA, electroacupuncture; MCAO, middle cerebral artery occlusion.
(Fig. 6B). These results suggest that EA may protect cortical cells against ischemic-induced apoptosis through the inhibition of the DR and mitochondrial apoptotic pathways.

Effects of EA on caspase activity. To further confirm the underlying signaling pathways mediating apoptosis, caspase activity assay was performed to assess the activation of caspase-3, -8 and -9 . The activities of caspase-3, -8 and -9 showed a significant increase in the rats with MCAO at $24 \mathrm{~h}$ after occlusion, compared with the sham-operated rats; these were markedly inhibited following treatment with EA (Fig. 7). These results confirm that EA may protect cortical cells against ischemic-induced apoptosis by inhibition the activity of caspases.

\section{Discussion}

EA has been widely used as a potential therapy for cerebral ischemia in clinical practice and basic research in Korea; however, the detailed mechanisms responsible for its beneficial effects remain unclear. In order to achieve clinical acceptance as an effective treatment for cerebral ischemia, an understanding of the mechanisms underlying the potent neuroprotective effects of EA is important. In this study, we first attempted to determine which region of the brain showed the most favorable neuroprotective effects by EA in a rat model of transient focal cerebral ischemia. We found that treatment with EA resulted in a significantly reduced infarct area, particularly in the middle region of the brain (Fig. 1).

We focused on the ischemic penumbra, which may be able to tolerate longer ischemic states compared with any other region; the effects of ischemia in this region may be reversible
A
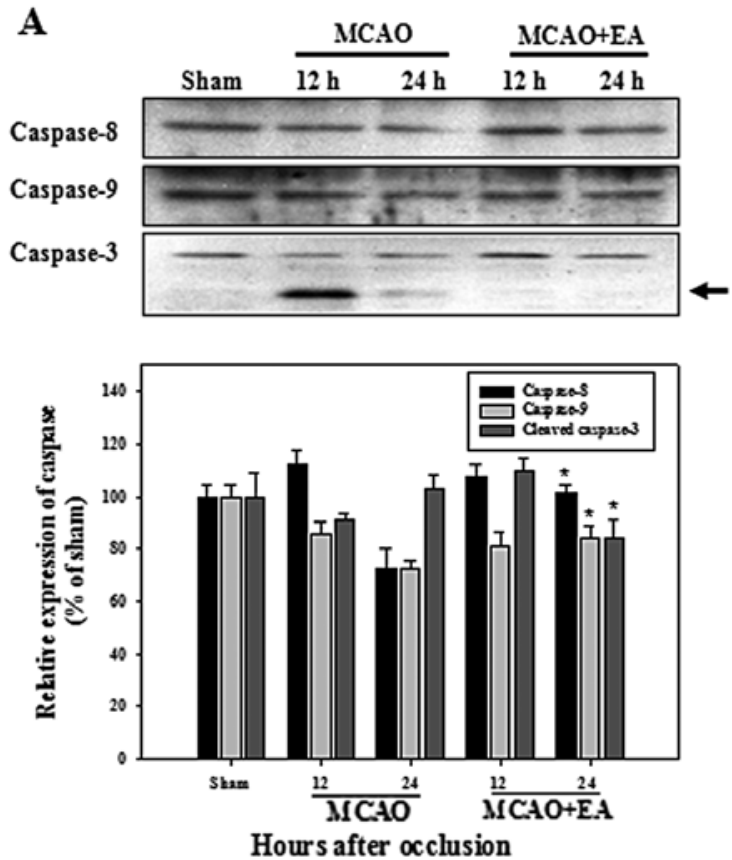

B
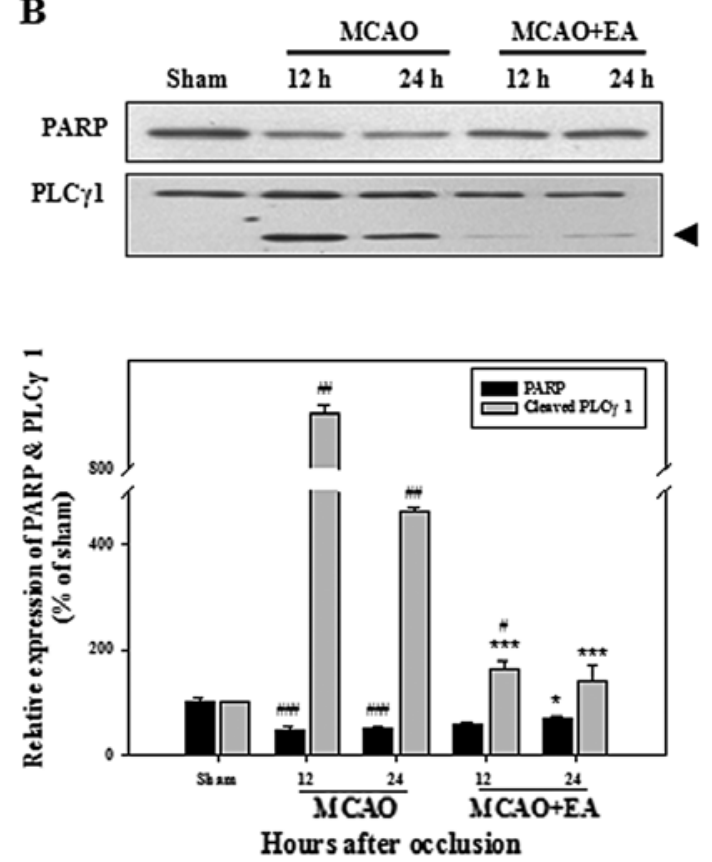

Figure 6. Western blot analysis and densitometric analysis for (A) caspases, (B) PARP and phospholipase C (PLC) $\gamma 1$ in the cerebral cortex of the brain. Each subunit is expressed as a percentage of the sham-operated rats. Representative results results from three independent experiments are shown. Cleaved (activated) caspase-3 (arrow) and cleaved PLC $\gamma 1$ (arrowhead) were markedly inhibited by treatment with EA. ${ }^{\#} \mathrm{p}<0.05$, ${ }^{\# \#} \mathrm{p}<0.01$ and ${ }^{\# \# \#} \mathrm{p}<0.001$ vs. sham-operated rats; ${ }^{*} \mathrm{p}<0.05$ and ${ }^{* * * *} \mathrm{p}<0.001$ vs. MCAO group rats; $\mathrm{n}=3$ in each group. EA, electroacupuncture; MCAO, middle cerebral artery occlusion. 
$\mathbf{A}$

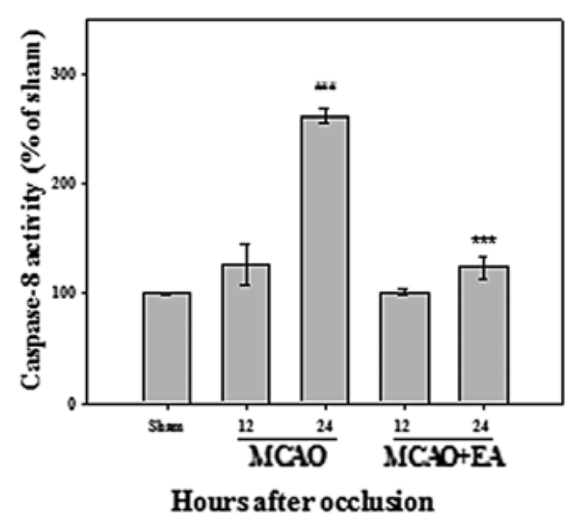

B

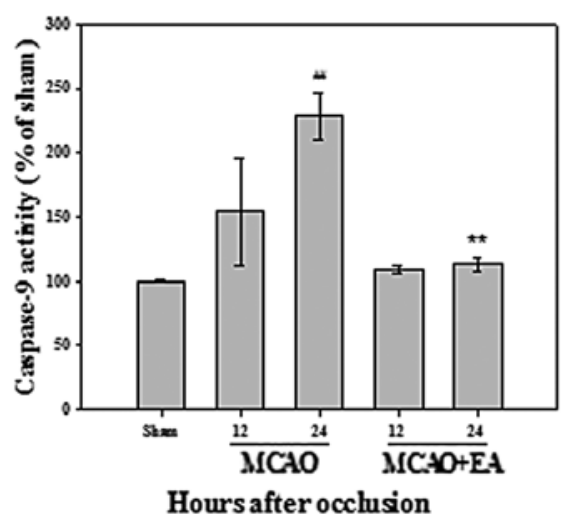

C

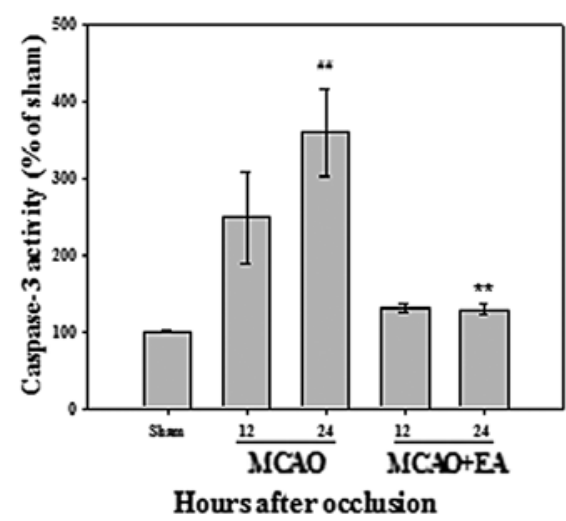

Figure 7. Caspase activity assay for (A) caspase-8, (B) caspase-9 and (C) caspase-3. The activities of caspase-8, -9 , and -3 in the MCAO group rats were markedly inhibited by treatment with EA. ${ }^{\# \#} \mathrm{p}<0.01$ and ${ }^{\# \# \#} \mathrm{p}<0.001$ vs. sham-operated rats; ${ }^{* *} \mathrm{p}<0.01$ and ${ }^{* * *} \mathrm{p}<0.001$ vs. MCAO group rats; $\mathrm{n}=3$ in each group. EA, electroacupuncture; MCAO, middle cerebral artery occlusion.

by a novel therapeutic strategy $(5,6)$. As opposed to the classical 'fried egg pattern', the ischemic penumbra is frequently embedded within the core (23); however, our morphological results revealed a similar apoptotic pattern in the middle cortical region of the brain. Chromatin condensation and TUNEL-positive cells can be used as evidence of apoptosis, and we confirmed that the ischemic penumbra contains apoptotic cells in the process of undergoing apoptosis; thus, it has the capacity for recovery. Apoptotic cells confirmed by Hoechst 33342 staining and TUNEL assay showed a significant increase after occlusion in the MCAO group rats, compared with the sham-operated rats; these apoptotic cells were markedly arrested by EA stimulation (Fig. 2).

In order to determine which factors can protect against apoptotic cell death of ischemic neurons, we analyzed related proteins and their activities occurring through DR and mitochondrial apoptosis. Death signals are initially induced by various pro-apoptotic stimuli and eventually converge into a mitochondrial-dependent mechanism activating death-execution caspases (24).

The analysis of the expression of death ligands and receptors revealed a significant upregulation of DR5, but not of TRAIL. The upregulated expression of DR5 and TRAIL, which occurs following transient ischemia-reperfusion, plays a deleterious role in the pathogenesis of delayed neuronal damage after global cerebral ischemia (25). However, in the present study, the upregulation of DR5 was markedly arrested by EA in the rats with MCAO (Fig. 3). This result suggests that the DR5-mediated death pathway may underlie hypoxicischemic neuronal apoptosis and that treatment with EA may result in the inhibition of DR5-induced apoptosis in cerebral ischemia.

The upregulation of DRs mediates caspase-8, initiator caspase-dependent neuronal death, and subsequently induces neuronal cell death in focal cerebral ischemia $(26,27)$. Activated caspase- 8 leads to the cleavage of Bid to the activated form, tBid, followed by the triggering of mitochondrial cytochrome $c$ release, which may eventually lead to the activation of caspase-3 $(28,29)$. In our study, we did not detect cleaved (activated) caspase- 8 and caspase- 8 cleaved Bid, as shown by western blot analysis. However, caspase- 8 activity was significantly inhibited by treatment with EA in the rats with MCAO (Figs. 6A and 7A).

The IAP family plays a major role in preventing apoptosis through the negative regulation of caspases. Members of the IAP family include cIAP-1, cIAP-2 and XIAP (30). All IAPs can potentially bind to caspases, but XIAP is the only member capable of blocking active caspases (31). cIAPs can regulate DR-mediated apoptosis, upstream of the mitochondria (32). DR-mediated apoptosis occurs through caspase 8-dependent cIAP-1 degradation in vivo (33). Although other caspases activated downstream of caspase- 8 may be involved in this pathway, as shown by our results, a significantly higher expression of cIAP-1 was observed in the rats in the MCAO + EA group, compared with the rats in the MCAO group (Fig. 5). These results suggest that treatment with EA may inhibit neuronal cell death through a DR-mediated apoptotic pathway, particularly by inhibiting DR5.

The involvement of the mitochondrial apoptotic pathway is supported by the release of cytochrome $c$ from the mitochondria to the cytosol in cerebral ischemia (34). Cytochrome $c$ in the cytosol catalyzes the oligomerization of apoptotic protease activating factor-1 (Apaf-1), which in turn promotes the activation of pro-caspase- 9 , leading to the activation of downstream pro-caspase-3, followed by apoptosis (35). An active role of the mitochondrial-mediated apoptotic pathway involving caspases after cerebral ischemia has been strongly suggested (11).

Caspases and Bcl-2 family members, along with members of the IAP family, appear to play critical roles in the regulation of multiple apoptotic cell death pathways during cerebral ischemia (4). The Bcl-2 gene family includes both anti-apoptotic Bcl-2 and Bcl-xL, as well as the pro-apoptotic proteins, Bax, Bak, Bad and Bid (4,36). Bcl-2 and Bcl-xL play a critical role in determining cell survival by inhibiting pro-apoptotic Bax $(37,38)$. In our study, the higher expression of the Bcl-2 family members, Bcl-2 and Bcl-xL, was observed in the rats in the MCAO + EA group (Fig. 4).

Activated caspase-9 can induce the activation of terminal caspase-3, which results in the mediation of the mitochondrial-dependent apoptotic pathway following cere- 
bral ischemia $(39,40)$. Caspase-3 is a major effector protease in cell death and cleaved caspase presents activation leading to cell death during ischemia $(15,41)$. XIAP plays a major role in the apoptotic process by directly inhibiting caspase- 9 , -3 and -7 (31); however, in this study, only a slight increase in XIAP expression was observed in the rats in the MCAO + EA group compared with the MCAO group rats (Fig. 5).

To further explore the underlying signaling pathways in mitochondrial apoptosis, we performed assays for the assessment of caspase activation and the cleavage of PARP and PLC $\gamma 1$. Neuronal cell death in stroke is associated with activated caspase-3 and with cleaved PARP and PLC $\gamma 1$ (4). The activities of caspase- 9 and -3 and activated caspase- 3 were markedly inhibited following treatment with EA in the MCAO group rats (Figs. 6A and 7). The inhibited activation of caspase-3 was accompanied by the reduction of cleaved PLC $\gamma 1$, a biochemical characteristic of apoptosis (Fig. 6B). Our results suggest that treatment with EA may also result in the inhibition of neuronal cell death through mitochondrial apoptotic pathways, with a higher expression of anti-apoptotic Bcl-2 and IAP family members in rats with MCAO.

We hypothesize that various signaling pathways may be involved in EA-induced neuronal cell survival. The phosphatidylinositol-3 kinase (PI3K)/Akt signaling pathway plays an important role in neuronal survival by protecting cells from caspase-mediated apoptosis and inhibiting pro-apoptotic proteins $(42,43)$. Our study demonstrated that EA possibly activates the PI3K/Akt pathway to initiate the cell survival pathway (44). EA may induce the activation of the PI3K/Akt survival pathway, possibly involving the anti-apoptotic form of the Bcl-2 and IAP families. Consequently, we conclude that the EA-induced neuroprotective effects against cerebral ischemia may be associated with the inhibition of two major DR and mitochondrial apoptotic pathways. Considering these results, treatment with EA may be an appropriate therapy against cerebral ischemia by controlling two main routes of the apoptotic pathway.

\section{Acknowledgements}

This study was supported by the R\&D program of MKE/KEIT (10040391, Development of Functional Food Materials and Device for Prevention of Aging-associated Muscle Function Decrease).

\section{References}

1. Shichita T, Hasegawa E, Kimura A, et al: Peroxiredoxin family proteins are key initiators of post-ischemic inflammation in the brain. Nat Med 18: 911-917, 2012.

2. Lansberg MG, Bluhmki E and Thijs VN: Efficacy and safety of tissue plasminogen activator 3 to 4.5 hours after acute ischemic stroke: a metaanalysis. Stroke 40: 2438-2441, 2009.

3. Li X, Luo P, Wang Q and Xiong L: Electroacupuncture pretreatment as a novel avenue to protect brain against ischemia and reperfusion injury. Evid Based Complement Alternat Med 2012: 195397, 2012.

4. Nakka VP, Gusain A, Mehta SL and Raghubir R: Molecular mechanisms of apoptosis in cerebral ischemia: multiple neuroprotective opportunities. Mol Neurobiol 37: 7-38, 2008.

5. Ginsberg MD: The new language of cerebral ischemia. AJNR Am J Neuroradiol 18: 1435-1445, 1997.

6. Love S: Apoptosis and brain ischaemia. Prog Neuropsychopharmacol Biol Psychiatry 27: 267-282, 2003.
7. Kato $\mathrm{H}$ and Kogure K: Biochemical and molecular characteristics of the brain with developing cerebral infarction. Cell Mol Neurobiol 19: 93-108, 1999.

8. Kerr JF, Wyllie AH and Currie AR: Apoptosis: a basic biological phenomenon with wide-ranging implications in tissue kinetics. Br J Cancer 26: 239-257, 1972.

9. Choi DW: Glutamate neurotoxicity and diseases of the nervous system. Neuron 1: 623-634, 1988.

10. Lee JM, Zipfel GJ and Choi DW: The changing landscape of ischaemic brain injury mechanisms. Nature 399: A7-A14, 1999.

11. Krajewski S, Krajewska M, Ellerby LM, et al: Release of caspase-9 from mitochondria during neuronal apoptosis and cerebral ischemia. Proc Natl Acad Sci USA 96: 5752-5757, 1999.

12. Lam TT, Abler AS and Tso MO: Apoptosis and caspases after ischemia-reperfusion injury in rat retina. Invest Ophthalmol Vis Sci 40: 967-975. 1999.

13. Xiong ZQ and McNamara JO: Fleeting activation of ionotropic glutamate receptors sensitizes cortical neurons to complement attack. Neuron 36: 363-374, 2002.

14. Neumar RW, Hagle SM, DeGracia DJ, Krause GS and White BC: Brain mu-calpain autolysis during global cerebral ischemia. J Neurochem 66: 421-424, 1996.

15. Namura S, Zhu J, Fink K, et al: Activation and cleavage of caspase-3 in apoptosis induced by experimental cerebral ischemia. J Neurosci 18: 3659-3668, 1998.

16. Broughton BR, Reutens DC and Sobey CG: Apoptotic mechanisms after cerebral ischemia. Stroke 40: e331-e339, 2009.

17. Campbell MT, Dagher P, Hile KL, et al: Tumor necrosis factor-alpha induces intrinsic apoptotic signaling during renal obstruction through truncated bid activation. J Urol 180: 2694-2700, 2008.

18. Wang SJ, Omori N,Li F, et al: Potentiation of Akt and suppression of caspase- 9 activations by electroacupuncture after transient middle cerebral artery occlusion in rats. Neurosci Lett 331: $115-118,2002$

19. Du Y, Shi L, Li J, Xiong J, Li B and Fan X: Angiogenesis and improved cerebral blood flow in the ischemic boundary area were detected after electroacupuncture treatment to rats with ischemic stroke. Neurol Res 33: 101-107, 2011.

20. Wang Q, Li X, Chen Y, et al: Activation of epsilon protein kinase $\mathrm{C}$-mediated anti-apoptosis is involved in rapid tolerance induced by electroacupuncture pretreatment through cannabinoid receptor type 1. Stroke 42: 389-396, 2011.

21. Sze FK, Wong E, Yi X and Woo J: Does acupuncture have additional value to standard poststroke motor rehabilitation? Stroke 33: 186-194, 2002.

22. Fang Z, Ning J, Xiong C and Shulin Y: Effects of electroacupuncture at head points on the function of cerebral motor areas in stroke patients: a PET study. Evid Based Complement Alternat Med 2012: 902413, 2012.

23. Foley LM, Hitchens TK, Barbe B, et al: Quantitative temporal profiles of penumbra and infarction during permanent middle cerebral artery occlusion in rats. Transl Stroke Res 1: 220-229, 2010.

24. Li P, Nijhawan D, Budihardjo I, et al: Cytochrome c and dATPdependent formation of Apaf-1/caspase-9 complex initiates an apoptotic protease cascade. Cell 91: 479-489, 1997.

25. Cui M, Wang L, Liang X, et al: Blocking TRAIL-DR5 signaling with soluble DR5 reduces delayed neuronal damage after transient global cerebral ischemia. Neurobiol Dis 39: 138-147, 2010.

26. Velier JJ, Ellison JA, Kikly KK, Spera PA, Barone FC and Feuerstein GZ: Caspase- 8 and caspase- 3 are expressed by different populations of cortical neurons undergoing delayed cell death after focal stroke in the rat. J Neurosci 19: 5932-5941, 1999.

27. Krupinski J, Lopez E, Marti E and Ferrer I: Expression of caspases and their substrates in the rat model of focal cerebral ischemia. Neurobiol Dis 7: 332-342, 2000.

28. Li H, Zhu H, Xu CJ and Yuan J: Cleavage of BID by caspase 8 mediates the mitochondrial damage in the Fas pathway of apoptosis. Cell 94: 491-501, 1998.

29. Luo X, Budihardjo I, Zou H, Slaughter C and Wang X: Bid, a $\mathrm{Bcl} 2$ interacting protein, mediates cytochrome c release from mitochondria in response to activation of cell surface death receptors. Cell 94: 481-490, 1998.

30. Wilkinson JC, Wilkinson AS, Scott FL, Csomos RA, Salvesen GS and Duckett CS: Neutralization of Smac/Diablo by inhibitors of apoptosis (IAPs). A caspase-independent mechanism for apoptotic inhibition. J Biol Chem 279: 51082-51090, 2004. 
31. Eckelman BP, Salvesen GS and Scott FL: Human inhibitor of apoptosis proteins: why XIAP is the black sheep of the family. EMBO Rep 7: 988-994, 2006.

32. Rothe M, Pan MG, Henzel WJ, Ayres TM and Goeddel DV: The TNFR2-TRAF signaling complex contains two novel proteins related to baculoviral inhibitor of apoptosis proteins. Cell 83: 1243-1252, 1995.

33. Guicciardi ME, Mott JL, Bronk SF, Kurita S, Fingas CD and Gores GJ: Cellular inhibitor of apoptosis 1 (cIAP-1) degradation by caspase 8 during TNF-related apoptosis-inducing ligand (TRAIL)-induced apoptosis. Exp Cell Res 317: 107-116. 2011.

34. Sugawara T, Fujimura M, Morita-Fujimura Y, Kawase M and Chan PH: Mitochondrial release of cytochrome c corresponds to the selective vulnerability of hippocampal CA1 neurons in rats after transient global cerebral ischemia. J Neurosci 19: RC39, 1999.

35. Earnshaw WC, Martins LM and Kaufmann SH: Mammalian caspases: structure, activation, substrates, and functions during apoptosis. Annu Rev Biochem 68: 383-424, 1999.

36. Yuan J and Yankner BA: Apoptosis in the nervous system. Nature 407: 802-809, 2000.

37. Oltvai ZN, Milliman CL and Korsmeyer SJ: Bcl-2 heterodimerizes in vivo with a conserved homolog, Bax, that accelerates programmed cell death. Cell 74: 609-619, 1993.

38. Sedlak TW, Oltvai ZN, Yang E, et al: Multiple Bcl-2 family members demonstrate selective dimerizations with Bax. Proc Natl Acad Sci USA 92: 7834-7838, 1995.
39. Kuida K, Haydar TF, Kuan CY, et al: Reduced apoptosis and cytochrome c-mediated caspase activation in mice lacking caspase 9. Cell 94: 325-337, 1998.

40. Noshita N, Sugawara T, Fujimura M, Morita-Fujimura Y and Chan PH: Manganese superoxide dismutase affects cytochrome $\mathrm{c}$ release and caspase-9 activation after transient focal cerebral ischemia in mice. J Cereb Blood Flow Metab 21: 557-567, 2001.

41. Shehadah A, Chen J, Zacharek A, et al: Niaspan treatment induces neuroprotection after stroke. Neurobiol Dis 40: 277-283, 2010.

42. Hsu AL, Ching TT, Wang DS, Song X, Rangnekar VM and Chen CS: The cyclooxygenase-2 inhibitor celecoxib induces apoptosis by blocking Akt activation in human prostate cancer cells independently of Bcl-2. J Biol Chem 275: 11397-11403, 2000.

43. Rau TF, Kothiwal A, Zhang L, et al: Low dose methamphetamine mediates neuroprotection through a PI3K-AKT pathway. Neuropharmacology 61: 677-686, 2011.

44. Kim YR, Kim HN, Jang JY, et al: Electroacupuncture confers beneficial effects through ionotropic glutamate receptors involving phosphatidylinositol-3 kinase/Akt signaling pathway in focal cerebral ischemia in rats. Eur J Integr Med 4: e413-420, 2012. 